\title{
Reference methodologies for radioactive controlled discharges an activity within the IAEA's program environmental modelling for radiation safety II (EMRAS II)
}

\author{
T.J. Stocki ${ }^{1}$, D.M. Telleria ${ }^{2}$, L. Bergman ${ }^{1}$, G. Proehl ${ }^{2}$, V. Amado ${ }^{3}$, I. Bonchuk ${ }^{4}$ \\ P. Boyer ${ }^{5}$, P. Chyly ${ }^{6}$, A. Curti ${ }^{3}$, R. Heling ${ }^{7}$, V. Kliaus ${ }^{9}$, P. Krajewski ${ }^{10}$, \\ G. Latouche ${ }^{11}$, D.C. Lauria ${ }^{12}$, C. Mourlon ${ }^{5}$, L. Newsome ${ }^{13}$, \\ L. Sági ${ }^{8}$ and J. Smith ${ }^{14}$ \\ ${ }^{1}$ Radiation Protection Bureau, Health Canada, 2720 Riverside Dr., AL6604C, Ottawa, \\ Canada, K1A OK9 \\ ${ }^{2}$ International, Atomic Energy Agency, Vienna, Austria \\ ${ }^{3}$ Autoridad Regulatoria Nuclear, Buenos Aires, Argentina \\ ${ }^{4}$ Ukrainian Radiation Protection Institute, Kyiv, Ukraine \\ ${ }^{5}$ Institut de Radioprotection et de Sûreté Nucléaire, Saint Paul-Lez-Durance, France \\ ${ }^{6}$ Slovak Electricity, Mochovce, Slovak Republic \\ ${ }^{7}$ Nuclear Research \& Consultancy Group, Es Arnhem, The Netherlands \\ ${ }^{8}$ KFKI Atomic Energy, Budapest, Hungary \\ ${ }^{9}$ Republican Scientific Practical Centre of Hygiene, Minsk, Belarus \\ ${ }^{10}$ Central Laboratory for Radiological Protection, Warsaw, Poland \\ ${ }^{11}$ Canadian Nuclear Safety Commission, Ottawa, Canada \\ ${ }^{12}$ Instituto de Radioproteçao e Dosimetria, Rio de Janeiro, Brazil \\ ${ }^{13}$ The Environment Agency, Warrington, UK \\ ${ }^{14}$ Health Protection Agency, Chilton, UK
}

\begin{abstract}
In January 2009, the IAEA EMRAS II (Environmental Modelling for Radiation Safety II) program was launched. The goal of the program is to develop, compare and test models for the assessment of radiological impacts to the public and the environment due to radionuclides being released or already existing in the environment; to help countries build and harmonize their capabilities; and to model the movement of radionuclides in the environment. Within EMRAS II, nine working groups are active; this paper will focus on the activities of Working Group 1: Reference Methodologies for Controlling Discharges of Routine Releases. Within this working group environmental transfer and dose assessment models are tested under different scenarios by participating countries and the results compared. This process allows each participating country to identify characteristics of their models that need to be refined. The goal of this working group is to identify reference methodologies for the assessment of exposures to the public due to routine discharges of radionuclides to the terrestrial and aquatic environments. Several different models are being applied to estimate the transfer of radionuclides in the environment for various scenarios. The first phase of the project involves a scenario of nuclear power reactor with a coastal location which routinely (continuously) discharges ${ }^{60} \mathrm{Co},{ }^{85} \mathrm{Kr},{ }^{131} \mathrm{I}$, and ${ }^{137} \mathrm{Cs}$ to the atmosphere and ${ }^{60} \mathrm{Co},{ }^{137} \mathrm{Cs}$, and ${ }^{90} \mathrm{Sr}$ to the marine environment. In this scenario many of the parameters and characteristics of the representative group were given to the modellers and cannot be altered. Various models have been used by the different participants in this inter-comparison (PC-CREAM, CROM, IMPACT, CLRP POSEIDON, SYMBIOSE and others). This first scenario is to enable a comparison of the radionuclide transport and dose modelling. These scenarios will facilitate the development of reference methodologies for controlled discharges.
\end{abstract}

\section{INTRODUCTION}

The International Atomic Energy Agency (IAEA) is conducting the Environmental Modelling for Radiation Safety II program (EMRAS II) to improve capabilities in the field of radiation dose 
assessment. This is accomplished by acquiring improved data for model comparison and testing. Then participants of the program reach a consensus on approaches, modelling philosophies, and parameter values. The overall purpose of the EMRAS II program is to exchange information and develop improved methods between international counterparts in the field of radiation protection.

The EMRAS II program builds upon previous international radioecological modelling exercises, namely BIOspheric Model Validation Study (BIOMOVS II), Validation of Model Predictions (VAMP), BIOsphere Modeling and ASSessment (BIOMASS), and the first EMRAS program. The BIOMOVS II program was initiated by the Swedish Radiation Authority. The remainder of the initiatives were sponsored programs of the IAEA.

The EMRAS II program emphasizes the improvement of environmental transfer models in order to reduce uncertainties and also the development of new approaches. This will improve the evaluation of radiological impact from radionuclides in the environment to humans and non-human biota. The EMRAS II program consists of three themes including reference approaches for human dose assessment, biota dose assessment and emergency situations, each theme is comprised of several working groups.

This paper is about the activities of Working Group 1: Reference Methodologies for Controlling Discharges of Routine Releases. The goal of this working group is to identify reference methodologies for the assessment of exposures to the public due to routine discharges of radionuclides to the terrestrial and aquatic environments and to identify the sources of differences in the results of the models. In the framework of this working group, several different models are being applied to estimate the transfer of radionuclides in the environment for various scenarios. In this paper, the preliminary results of one of these scenarios are discussed.

\section{SCENARIO MODELLED AND MODELS USED}

\subsection{Scenario A - Sizewell B, United Kingdom}

This scenario involved a reactor on a marine coast. This scenario was partially based on the Sizewell B nuclear power station located on the east coast of England, United Kingdom. The Environment Agency of England and Wales has already published its own radiological assessment for Sizewell B, based on the actual discharge data [1]. A habits survey for the communities surrounding Sizewell was completed in 2005 [2]. Information from these published reports was used to develop Scenario A. The benefit of using data from an actual nuclear power station is that the scenario is realistic, and readily available published data can be used to facilitate the model inter-comparison exercise.

Sizewell B is a pressurised water reactor located on the coast of Suffolk, England. It came into operation in February 1995. Sizewell B is co-located at the same site as Sizewell A, which has twin Magnox reactors. Sizewell A ceased generating electricity at the end of 2006.

The Sizewell site is located about $3 \mathrm{~km}$ from the villages of Leiston and Thorpeness. Authorised discharges are made to atmosphere via stacks on the site (effective height $19 \mathrm{~m}$ ), and to the North Sea via pipelines. This data was used in Scenario A.

For comparison purposes, a hypothetical radiological inventory of discharges was created. It was assumed that $1 \mathrm{TBq} / \mathrm{y}$ of $\mathrm{Kr}-85$, Co-60, I-131 and Cs-137 are discharged to air, and $1 \mathrm{TBq} / \mathrm{y}$ of Cs-137, Co-60 and Sr-90 are discharged to the North Sea. These release levels are merely unit discharges for model inter-comparison reasons, and do not represent the actual Sizewell releases. The remainder of the scenario was based on environmental conditions and habits from real data [2].

\subsubsection{Parameter Standardization}

To ensure quality control, templates were set up with standardized parameter values. This was done to focus the interest on the differences between the models themselves, and not on different choices of parameters or on different interpretations of the available input information. (Early results had shown 
that there were large discrepancies between models when a standard set of parameters was not used). As much as possible the parameters were taken from the literature [2-7].

\subsection{Software Packages and Models used for Scenario A by the modellers}

There are various models in the literature that can be used for modeling the transfer of radionuclides in the environment. One example is the Gaussian plume model [8]. These models can be simply calculated in a spreadsheet (modeller 7 used a spreadsheet according to the models in [4]) or by using an available software package. The software packages used in this project are: PC-CREAM (98 and 08 versions), IMPACT, CROM, SYMBIOSE, CLRP, POSEIDON (POSEIDON-R (RODOS)), and RDEMO [3, 4, 9-15]. Below are brief descriptions of some of these software packages. All of these packages are radionuclide environmental transfer models, considering a number of different transfer pathways. The goal of each of these packages is to determine the dose to a human receptor. Depending on the criteria used to define receptor dose, this person could be a critical group of people for which the dose would be considered the highest, or it could be a representative person.

\subsubsection{PC-Cream (modellers 1,4 and 5)}

PC-CREAM 08 is a complex model divided into different components for ease of use. The program is divided into 'Models' and 'ASSESSOR' components. The Models component includes a series of mathematical models that predict the transfer of radionuclides through the environment and provide estimates of activity concentrations in various environmental media following a continuous release. The output of these models is then used as input to the dose assessment part of the program ASSESSOR. PC-CREAM 08 is capable of modelling discharges to the atmosphere, European coastal waters and rivers to determine both individual and collective doses. More complete details pertaining to the models and data are given in the methodology report [9]. This study also included estimates of dose made using the previous version of the software, namely, PC-CREAM 98 [10] (modeller 5).

\subsubsection{IMPACT/N288.1 (modeller 2*)}

The software IMPACT is based on the Canadian Standards Association standard N288.1 [3]. The N288.1 standard is used to calculate derived release limits (DRL), but can equally be used to calculate annual public doses to humans through the use of mathematical models which represent the transfer of radioactive material through the environment to humans. This model takes into account all exposure pathways, which includes external exposure from immersion in contaminated air and water; exposure to contaminated soil and beaches; and internal exposure from inhalation and ingestion. The standard covers releases to the atmosphere and to surface water (fresh and marine) and only addresses routine, low-level emissions. The human receptor considered in this standard is a representative person having the average characteristics of a group of individuals who are likely to receive the highest exposures to a given radionuclide released. In terms of atmospheric dispersion, the standard is only valid up to $20 \mathrm{~km}$ from the facility and does not apply to people located so close to a source that building-induced turbulence is an effect.

\subsubsection{CROM (modeller $\left.9^{*}\right)$}

CROM is a generic environmental model code developed by the CIEMAT (Centro de Investigaciones Energéticas, Medioambientales y Tecnológicas) in collaboration with the Polytechnic University of Madrid. It is based on IAEA Safety Report Series 19 [4], with some variations from RP 72 [10]. CROM

*Modifications of the software or supplementary calculations outside of the software were necessary. 
uses a Gaussian plume model to assess annual average radionuclide concentrations in air and the rate of deposition, validated for distances $<20 \mathrm{~km}$. The model accounts for the effects of any buildings in the vicinity of the release. The basic meteorological variables required are the wind direction and the geometric mean of the wind speed at the physical height of the release point; however, the height resulting from the rise of the effluent plume owing to thermal or mechanical effects is not considered. The code allows the use of other diffusion factors, for different Pasquill stability categories [8] other than class $\mathrm{D}$, and effective heights but does not calculate them. The terrestrial food chain models accept inputs of radionuclides from both the atmosphere and the hydrosphere and it takes account of the buildup of radionuclides on surface soil over a 30 year period. The process of radioactive decay and build-up is taken into account in the estimation of the retention of radionuclides on the surfaces of vegetation and on soil, and in the assessment of the losses owing to decay that may occur during the time between harvest and vegetable consumption. The food categories considered in the model are milk, meat and vegetables. The uptake and retention of radionuclides by aquatic biota uses selected element specific bioaccumulation factors that describe an equilibrium state between the concentration of the radionuclide in biota and water. The types of aquatic food considered are freshwater fish, marine fish and marine shellfish. The estimated radionuclide concentrations in air, soil, sediment, food and water (representative of 30 years of discharge) are combined with the annual rates of intake and the occupational factors and the appropriate dose conversion coefficients to obtain the maximum effective dose in one year for the combined external and internal exposure.

\subsubsection{SYMBIOSE (modeller 3)}

SYMBIOSE is a simulation platform for assessing the fate and transport of pollutants in environmental systems, and their impact on humans [11]. Featuring a flexible and modular architecture, it deals with a wide range of situations, from simplified generic studies to spatially-distributed, site-specific assessments. Co-funded by IRSN (Institut de Radioprotection et de Sûreté Nucléaire, France) and EdF (Electricité de France), it is to be a reference tool for assessing doses induced by radioactive releases from nuclear facilities under accidental, decommissioning or normal operating conditions.

Atmospheric, terrestrial, freshwater and marine systems are modelled, and major transfers between them. When possible, alternative modelling approaches, of varying complexity, are provided. Specific models are dedicated to hydrogen, carbon and chlorine. A spatial pre-processor produces sitespecific landscapes from geographical database information. The calculations are performed by an engine offering various numerical solvers dealing with possibly complex system dynamics, in either deterministic or probabilistic mode.

\subsubsection{POSEIDON-R (modeller 8)}

The Poseidon $-\mathrm{R}$ model is a modified version of the original POSEIDON-PC software for dose assessments for discharges to the marine environment, from both atmospheric fallout and direct discharges. The direct discharges are from rivers, land-to-water, or from direct releases from a nuclear power station. POSEIDON is a component of the Realtime online decision support system for nuclear emergencies RODOS. The module for the uptake of radionuclides by marine organisms (BURN) is dynamic and based on prey-predator - relations. This avoids the use of concentration factors (CF) from the literature, which are often conservative, and gives realistic concentrations of radionuclides in biota $[13,14]$. The module is salinity driven for caesium and strontium uptake [14]. BURN is a physiological model where the uptake behaviour is determined by the tissue in which the radionuclide accumulates. At present the dominant pathway of the model is for the dose assessment for the consumption of seafood. This software package was only used to model the marine portion of the scenario. 


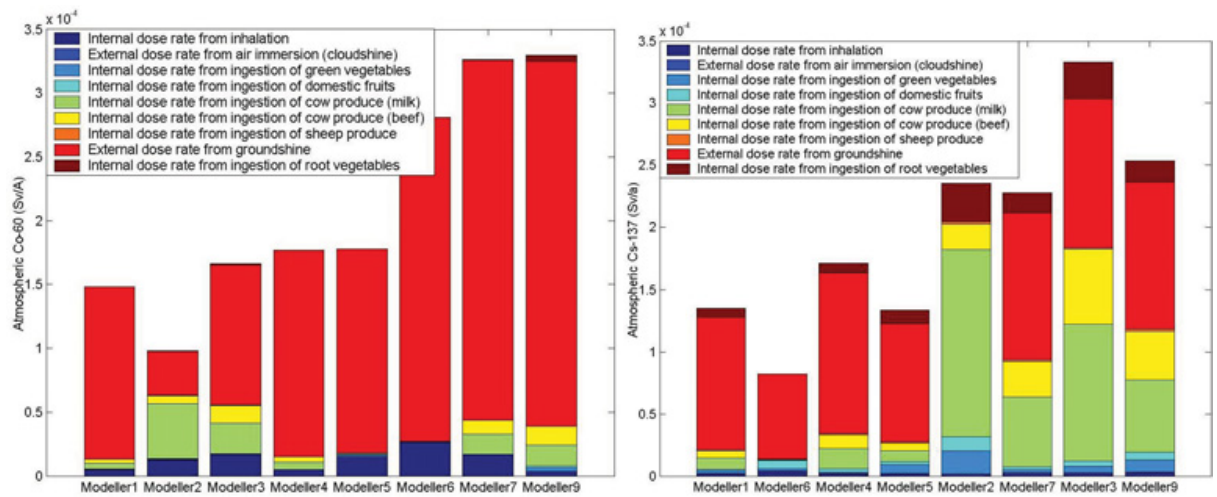

Figure 1 (left) and Figure 2 (right). The dose calculated to a person from the Sizewell scenario critical group, from a hypothetical release of Co-60 (left) and Cs-137 (right) of $1 \mathrm{TBq}$ to the atmosphere.

\subsubsection{RDEMO (modeller 6)}

The code RDEMO is deterministic computing program model used for the estimation of the radiological consequences from radioactive discharges into the atmosphere and hydrosphere from nuclear power plant Mochovce during normal and abnormal operation. The mathematical model of the transfer of radioactive substances to humans and their dose uses a compartment model based on the concentration coefficient method. The following radiation pathways are considered: atmosphere, hydrosphere and food chains. Calculation of the dispersion of substances in atmosphere uses the Gaussian model of atmospheric diffusion. The calculation in hydrosphere only considers surface water effects. Input parameters for calculation consist of local data. The program enables calculation for six age categories, for six calculated body organs and for the whole body and for ten radiation exposure pathways. It also calculates regional doses. The program determines the critical zone, critical population group, critical exposure pathway and critical radionuclides for individual exposure pathways. Program set RD (Radiological Doses) was developed by VUJE Trnava for nuclear power plants in Slovak republic and Czech Republic. The RD code has been validated by Expert Commission of SUJB Czech republic. Moreover, RDEMO has been validated also by comparison with the code NRC Dose.

\section{RESULTS}

To achieve consistent and comparable results for Scenario A, it was very important that all the modellers use the same input parameters or compatible ones. There have been a number of iterations between obtaining results and setting compatible parameters in order to reach a set of standardized parameters. This was done in order to address discrepancies in the results that were attributable to the use of parameters and not truly reflecting differences in the models.

The majority of models used a Gaussian plume [10] model to predict atmospheric dispersion and consequently activity concentrations in air were broadly similar i.e. within $30 \%$. Figure 1 shows the results from each of the models for the atmospheric release of Co-60. In this graph the results are broken down into different components (via different exposure pathways) of the total individual dose for the critical group. In general, the results only differ by a factor of $\sim 4$ between different models. It is interesting that the different models returned different values for the groundshine exposure pathway. Also for some models a significant component of the dose was due to milk products, whereas other models did not find a significantly large amount of dose from milk products, even though the Co-60 concentration in air was found to be about a 30\% difference between models. For instance the older version of PC cream did not have a value for radiation dose from milk, whereas the new version did. 


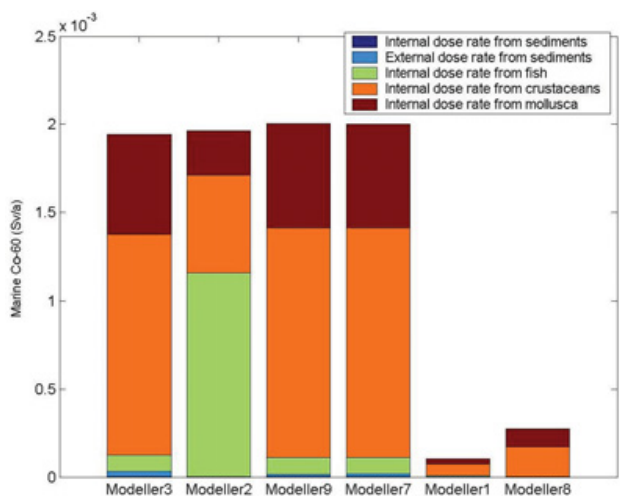

Figure 3. The dose calculated to a person from the Sizewell scenario critical group, from a hypothetical marine release of $1 \mathrm{TBq}$ of Co-60.

Similarly, Figure 2 shows the results for a modeling the dose to a human receptor from the atmospheric release of Cs-137. In general the results are within a factor of $\sim 4$ of each other. Again the graph is broken down by the different exposure pathways. In contrast to the Co-60 results, the Cs-137 results have larger differences by each pathway. For example the external dose rate from the groundshine exposure pathway is very significant in some models, but insignificant in others. Similarly, for the internal dose rate from beef, ingestion was found to be significant in only some of the models.

Figure 3 shows the results from the models for marine release of Co-60. This time the results are not within a factor of 4 , like the atmospheric results. The results can be visually divided into two distinct groups with fairly close agreement within each group. This is due to the fact that one group of models which are plume based models and the other group of models are box models. For example, in some of the codes the dispersing radionuclides are modelled using a Gaussian plume and it is conservatively assumed that the fish and shellfish for consumption are caught from the centre line of the plume. However, in others using a box model, such as that used in PC-CREAM 08, instant dilution into the local box around the site is assumed and hence for this particular scenerio the model is less conservative, in this case by a factor of about 20 . The size of the local box will affect this difference as well as the selection of the exposure scenario. Furthermore one of the box-models used a dynamic food model, without use of fixed concentration factors, resulting in less conservative, but realistic, uptake behaviour.

\section{CONCLUSION}

The goal of this exercise is not to find the best model, but however to give a benchmark for people to use when they start environmental modelling. To conclude, the various radionuclide environmental transfer models used by different modellers were inter-compared for the scenario of a reactor located near a sea coast. The results for an atmospheric release of Cs- 137 and Co-60 varied within a factor of 4 . This is reasonable considering the complexity of the physical and chemical processes that govern the movement of radioactive material in the environment and the inherent natural variability that can occur. Considering that each result was done by a different modeller, this is satisfactory agreement. For a marine release of Co- 60 , the results are more interesting. There is about a 20 times difference between box models and plume models discovered in the modelling of this specific scenario. This is a very interesting and significant result, because it can give guidance to future modellers on the level of caution built into the models and provide input to decisions on the type of models to use.

Further details of the scenario and analysis of the results from the Sizewell B scenario is underway and will be presented in the EMRAS II IAEA final report. In this report, Working Group 1 will 
also be completing and presenting the results from the modeling of a river scenario based on Chalk River, Canada.

\section{References}

[1] Punt, A., Kruse, P. and Titley, J. G., Radiological assessment - British Energy Sizewell B. Authorisation review 2005/6, post-consultation amended version. Environment Agency report reference NMA/TR/2006/05a (2006).

[2] Clyne, F. J., Tipple, J. R. and McTaggart, K. A., Radiological habits survey: Sizewell, 2005. Cefas report reference RL02/06 (2006).

[3] Ryan J. et al. Guidelines for calculating derived release limits for radioactive material in airborne and liquid effluents for normal operation of nuclear facilities. Canadian Standards Association document N288.1-08. Mississauga (2008).

[4] International Atomic Energy Agency Generic Models for Use in assessing the Impact of Discharges of Radioactive Substances to the Environment, IAEA Safety Series No. 19, IAEA Vienna (2001).

[5] Brookhaven National Laboratory. National Nuclear Data Centre NuDat 2 website http://www.nndc.bnl.gov/nudat2

[6] International Atomic Energy Agency Handbook of Parameter Values for the Prediction of Radionuclide Transfer in Temperate Environments. Technical Report Series 364 IAEA Vienna (1994).

[7] Stocki, T.J. et al. Radioprotection 44 (2009) 735-739.

[8] Pasquill, F. Atmospheric Diffusion: The dispersion of Windborne Material from Industrial and other sources, Van Nostrand Co., London (1962).

[9] Smith, J.G. and Simmonds J.R. (editors). The methodology for assessing the radiological consequences of routine releases of radionuclides to the environment used in PC-CREAM 08, HPA-RPD-058 (2009).

[10] Simmonds, J.R, Lawson G., and Mayall A. Methodology for assessing the radiological consequences of routine releases of radionuclides to the environment. Radiation Protection 72. Report EUR 15760 (1995).

[11] Gonze M.A., Garcia-Sanchez, Boyer P., Mourlon C., Tamponnet C. SYMBIOSE: A modeling and simulation platform for environmental chemical risk assessment. Proceedings of the Third International Symposium on the Protection of the Environment from Ionising Radiation (SPEIR 3) held in Darwin, Australia, 22-26 July 2002. ISBN 92-0-103603-5. IAEA 2003, pp. 266-274.

[12] Lepicard. S., Heling R., Maderich, V. (2002) POSEIDON/RODOS for radiological assessment of marine environment after accidental releases: application to coastal areas of the Baltic, Black and North Seas. Proceedings of International Symposium on "on situ Measurements as a tool for radioecology. IRE Conference, Fleurus, Belgium, 10-12 June 2002

[13] Heling R. Koziy L. Bulgakov V. Radioprotection 37 (2002) C1-833-C1-838.

[14] Heling, R, Bezhenar R. Radioprotection 44 (2009) 741-746.

[15] Krajewski P., et al. Int. J. Radiat. Appl. Instrum. Part A 66 (2008) 1730-1735. 